\title{
Pathway Discover of Moving Objects using Color Segmentation and Region property
}

\author{
Amany Mohammad Abood ${ }^{1}$, Zainab G. Faisal ${ }^{2}$, Maysam Sameer Hussein ${ }^{3}$ \\ ${ }^{1-3}$ Computer Engineering Techniques, \\ Al-Esraa University College, Baghdad, Iraq \\ 1 amany@esraa.edu.iq \\ 2 zainab.ghazi@esraa.edu.iq \\ 3 maysam@esraa.edu.iq
}

\begin{abstract}
This paper presents the real time algorithm of moving objects tracking and detection using region property and color segmentation. The real time of moving objects pathway finder is a vitaldifficultyproblem in human computersinterface and video observation. The attitude of track and detect a moving objects using color characteristic and movements has introduced with new techniques for automation. The tracking of video is a method of discovery the travelthing over specific reserve by use a color camera to narrate target bodies in successive video borders. Respecting to frame rate, the relationship could be especially troublesome in case of speedy moving of objects.In interchange case, the issue grows of randomness is the time in case of the tracking objects varying the direction following eventually. For this cases, the video tracking design model are classicallyexploit the progress model willportrays process the image of the target when it CHANGE for characteristicimaginableobjects movements. A development of tracking algorithm to the real time affecting bodies with unlike frames of the videotape by use color characteristic and movement is investigated and produced in this work.
\end{abstract}

Keywords - Pathway Discover, Moving Objects, Color Segmentation, Region property

\section{INTRODUCTION}

The tracking technology could be typified as a difficulty of evaluating the route of anthings in the image as it shiftsroughlyat the sight [1]. The convenience of high excellence and high power computers requires for automaticcharacteristic investigation has formed a many concernpathway algorithms of moving objects [2]. The important key in characteristicexaminationof moving body's surveillance is:

a. Moving objects recognition

b. Moving objects tracking starting frame to another frame

c. examination of things tracking

The diversity of existingattention in PCapparition need aability to track moving bodies in life stream to video conference or robot direction-finding [3]. The complexities in force a hugeelement of investigation in this topic are the huge informationmoveability incidental by good declaration frames with elevated frame rate [4]. The enormous majority of the routines which use complex model such as formatting is a computationally Eigen. In addition, the detail of these methods is expensive in the capacity offerdependable object credit [5]. At high frames rate, the tracking are extremely not talented to sprint on occupied video declaration image [6]. In general, the utilization of color is a real time pathwayborder works which offer a littlenotable points of importanceabovearithmetic signs as in heftinessbelowfractional occlusion and computational simplicity [7]. The color blob is tracked in the framework of tracking systems [8]. Theblob idea is represents the image individualityaextendednarration in the apparition of computer which is numerically define in many definitions [9]. Minimum pixels that are pass on illustration possessions which are absolutely not important by adjacent pixels [10]. The analysis of object movement is avitalnovelty to mergecurrentbio procedure associated with the vision of computers [11]. In general, it is utilize as branch of intellectual, interaction of human mechanism, analysis of association and a lot of others field [12]. Currently, the systems are exploiting as aelement of affecting object detection and tracking to provide frame subtraction and optical flow methods with background subtraction policy [13]. The region of interest (RIO) is the most important area and commonly recognizes issue in the body tracking [14]. The RIO is the setting of finding the necessary body in individual frame of video and requiresdetecting the movement of this body with support of movement opinioncharacteristic [15]. In addition, the light illumination and background is the problem which materializeamong the body tracking by noise and need some filtering techniques to eliminate out of interest region [16]. The major difficulties in the body tracking are the noise, complex objet shape and movement, body occlusions, sight illumination and continuous processing [17]. The motiondiscovery by usingenvironmentcalculationscheme has proposed by [18]. They progress the video image which consist the feature of geometry information of the target and divide relevant analysis of target movement. The description of movement detection using new algorithm based subtraction of background is introduced by [19]. They design a model based on statistical utilization and dependable 
background. In this suggestion, the calculationamongpresent image and backdrop image has carry out depend on doorsill level and they use a morphological filter to remove the noise associated with the process. The movement detection has been displayed by overcome the drawback of background subtraction algorithm in [20]. They compute the background subtraction algorithm and utilize the capability to determine the matter of restrictedlighting change. The movement detection and utilizing optical flow policy by [21] is come out from relationsociety of body and the watcher to provideserious information about the spatial collection of body with rate change of the object position. The development of motion detection by use the arrangement depends on sequential disparity and visualstream has produced by [22]. The background frame matching of movement detection has proposed by [23]. In this technique, they extremely effective for seem at picture pixel in resulting unmoving frames imprisonfollowingnormal time from camera. They oblige two borders to discover body movement in his proposal one is frame identifylocation frame and the other called input frame which contain the objet in moving phase. These two frames have examined and division in pixel character is determined.

The main objective in this paper is to track moving objects using individual frames associated with suggested algorithms to carry out video tracking algorithm. The video features and frames has been investigated using different algorithms considering the utilization is essential which selection algorithm is utilize. The proposed system will produce a variety of tools to recognize the movement of the objectives. The proposed algorithm has been improved to track the moving objects and implemented in MATLAB. The basic blocks to discover and track the moving target using color feature and motion is demonstrated in Figure 1.

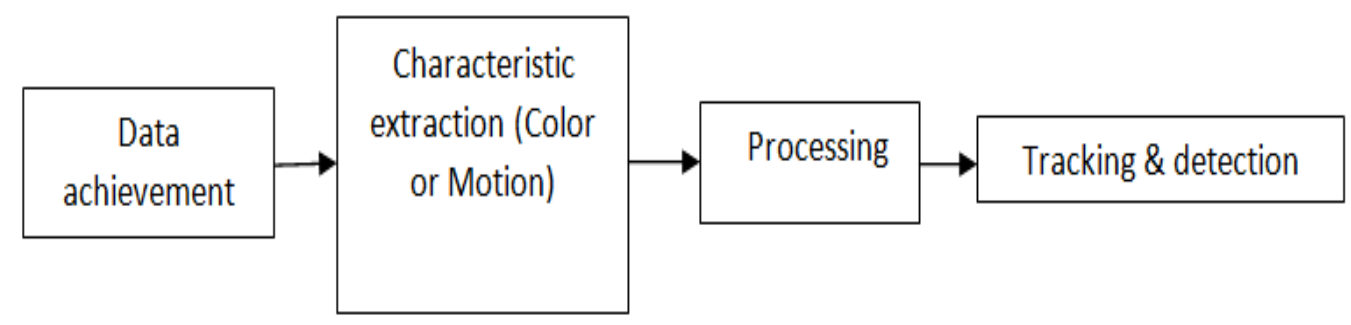

Fig. 1.Basic block diagram of moving objects tracking

The data achievement proposes to acquire the captured frames using thepicture process tools in which are gained with camera show in the frameworks. The processing step is changed the picture color into gray color on the ground which is easy to make a processing in single shade. The gray color is obligate less time in treatment. Then, the filtering process is applied to eject clamor from image frames provided from the video. The command (medfilt) has been used to analyze the frames from image processing tools. Characteristic or features extraction step is for choice the correct feature which the important step in the tracking technique. These features such as color, optical flow, edge and surface represent vital key in the tracking algorithms. The red, green and blue colors is utilize in the proposed approach which is simplify capable to track a moving objects. The motion detection phase use different frame methods with optical flow techniques. The tracking step carry out on the premise local specifications of the object such as box bounding, area and centric to utilize the track process.

\section{SUgGeSTED Algorithm}

Figure 2 illustrate the suggested algorithm for object tracking and detection using color segmentation and region property. To capture the frames from video, the camera is used for image processing from video input based on video achievement purpose which is specify the specifications of property in the video input. The video acquisition started after specific number of frames to create loop in the subsequentladder:

a. Capture a picture of the initial frame from the videocassette

b. pathway the current color (red) of the bodies and subtract it from gray color

C. Run the removal noise of designed filter

d. Change any gray levelpicture to binary picture

e. Eliminate all pixel less than 400 pixels

f. Implement image blob to all segments

g. Exhibit the image and used the loop once more to bounce red color object in rectangular form.

h. discontinue any enter from camera video

i. Delete all information from the memory and flush all variable 


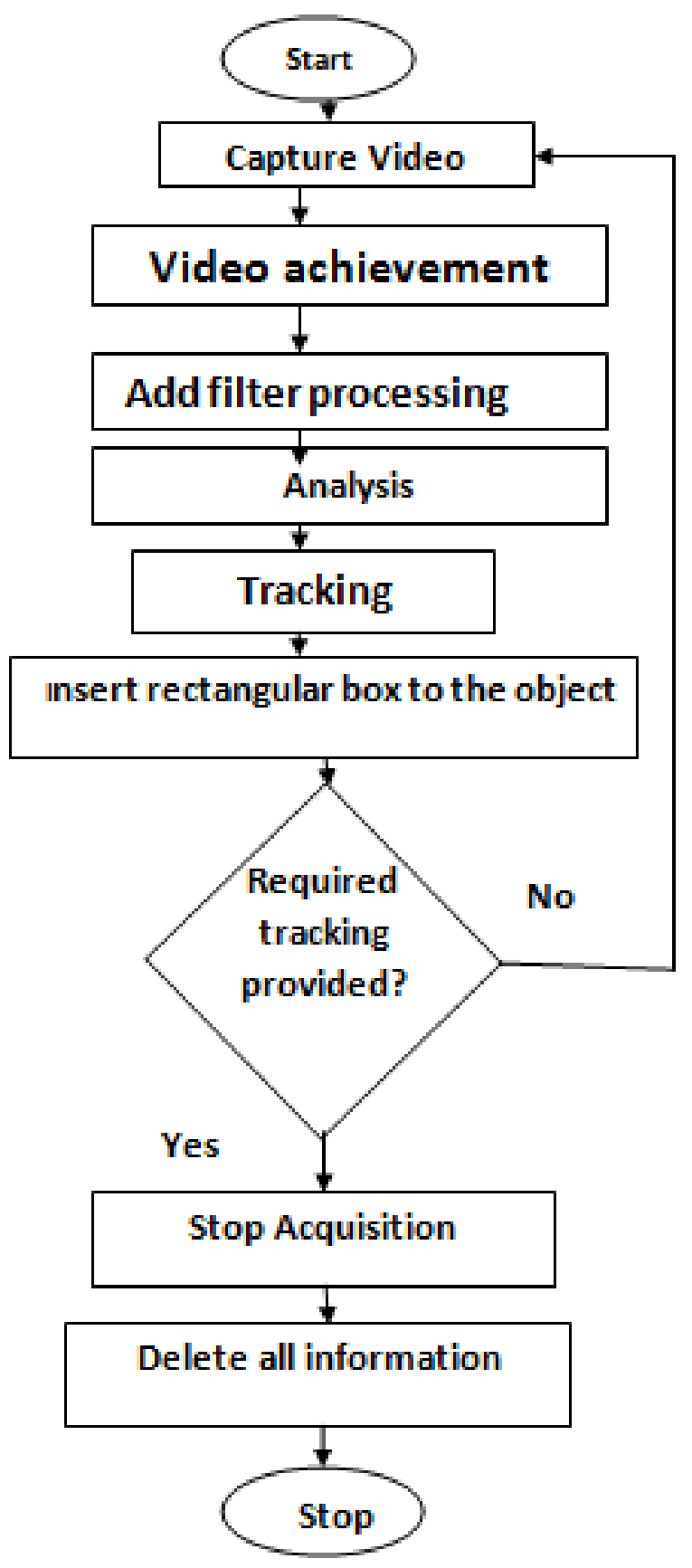

Fig. 2.Projected algorithm for discovery and tracking moving bodies

III. MatLab Model

Figure 3 shows the model based design of detection and tracking for moving objects depend on color information. The color based tracking model shows how to use the color space conversion block set. 


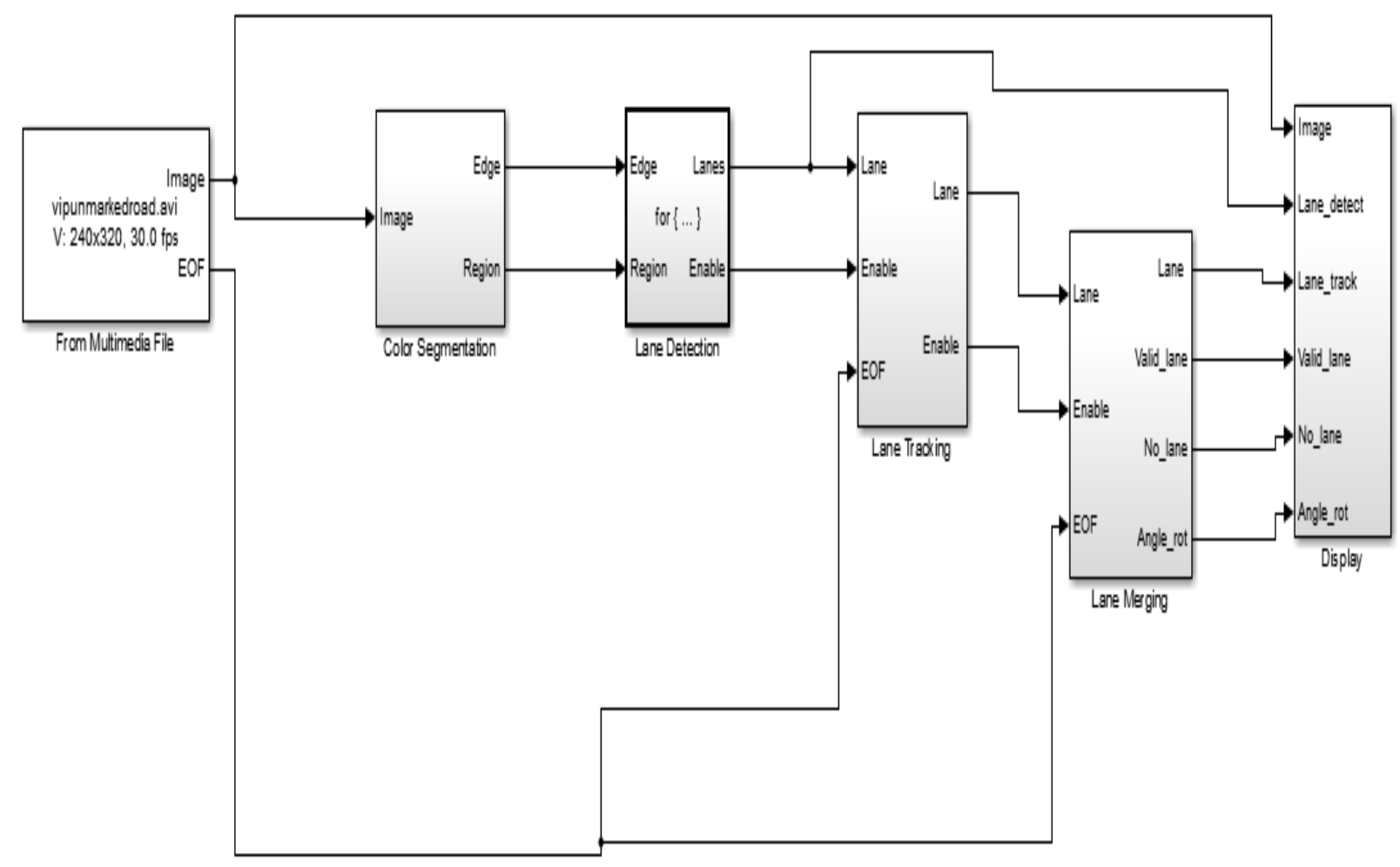

Fig.3. Color based tracking and detection of moving objects using MATLAB

The subsystem of each block is illustrated in Figure 4, 5, 6, 7 and Figure 8. The color segmentation subsystem model in this algorithm is used to perform aninvestigation to describe the right and leftboundaries of street based analysis the video images for color behavioral changing. Firstly, the search for edge pixel or line passing via enough number of color pixels either comes first is started from bottom center of the image which progress togetherthe upper left and right bend.

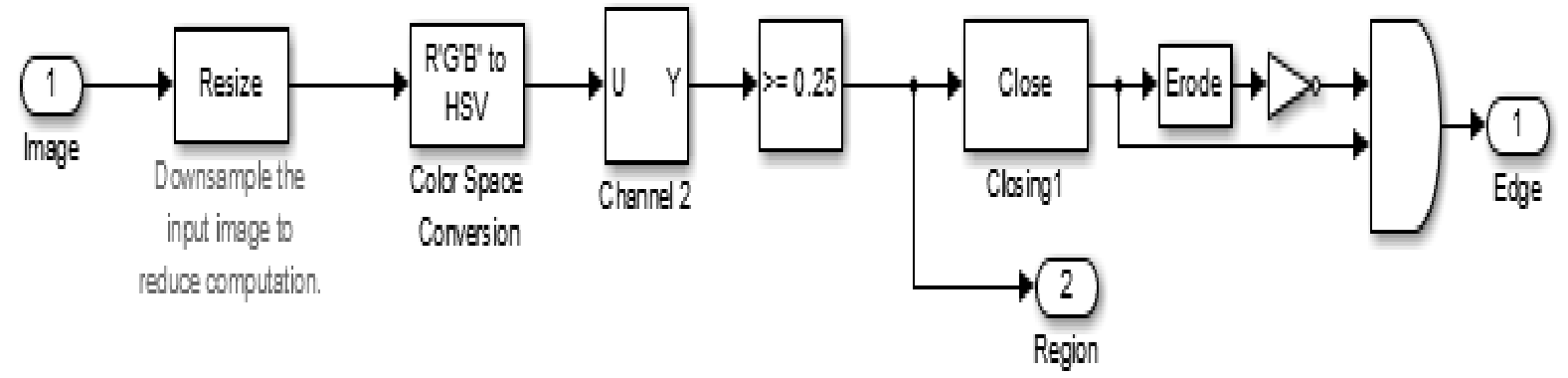

Fig. 4. Color segmentation subsystem model 


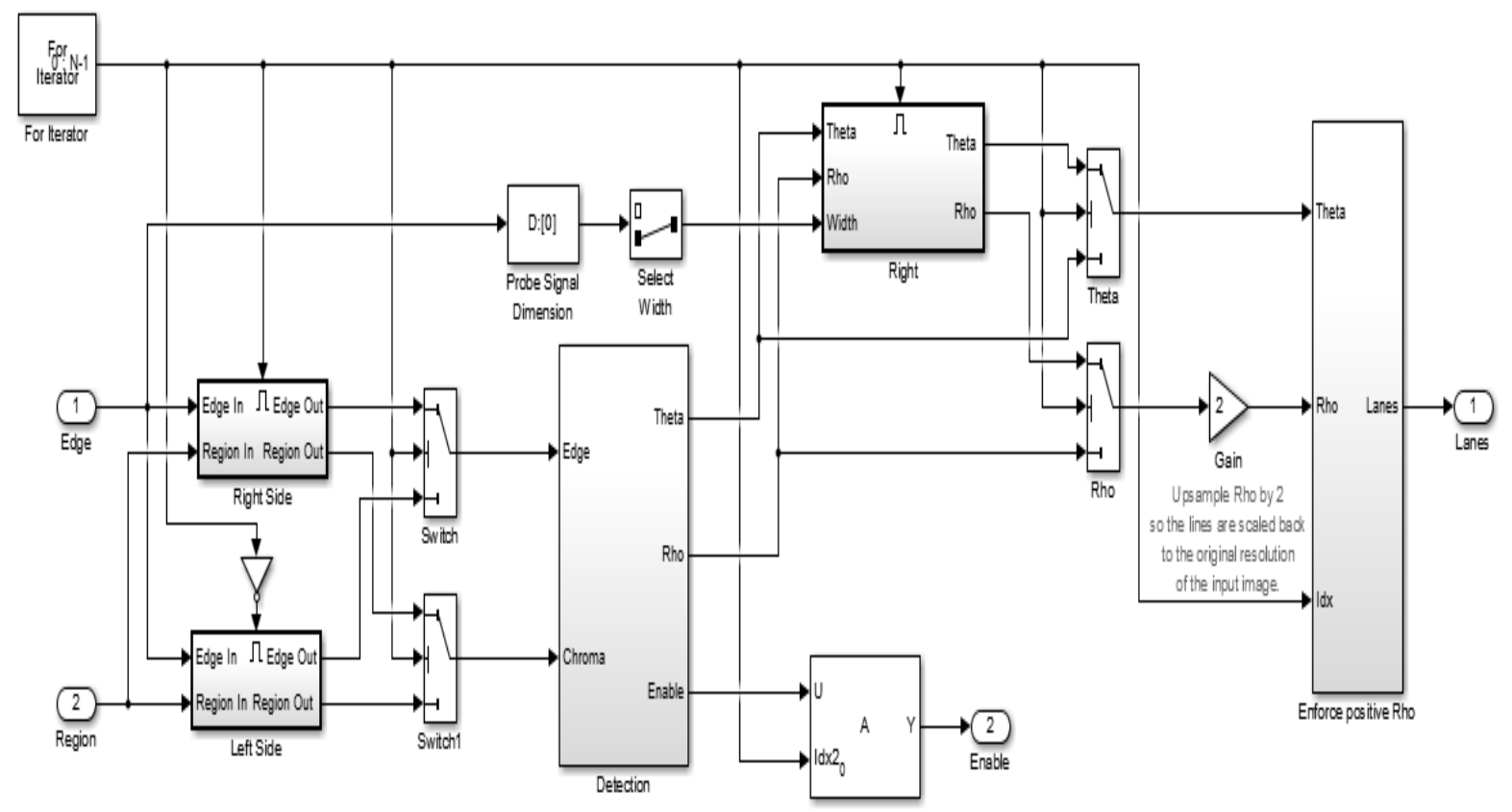

Fig.5.Lane detection subsystem

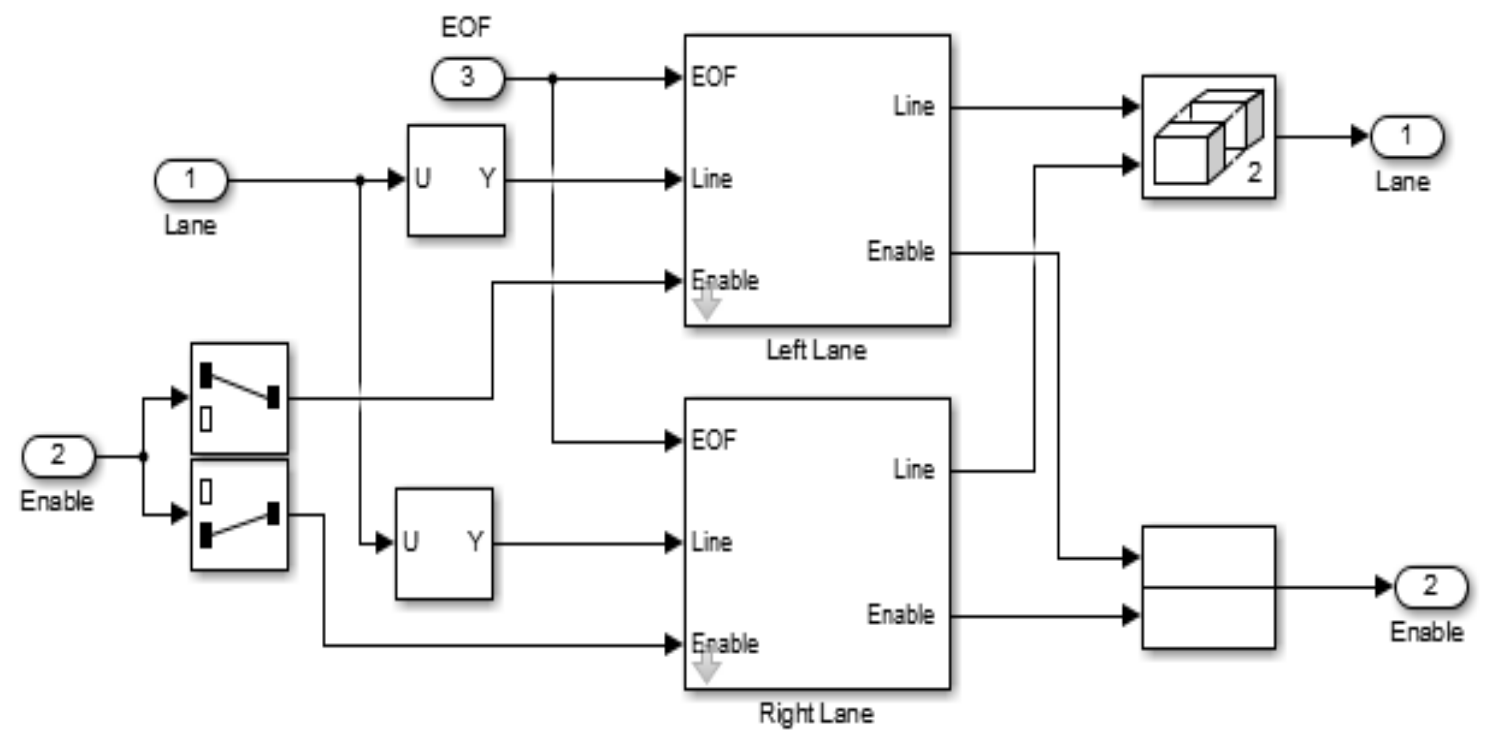

Fig.6. Lane tracking subsystem 


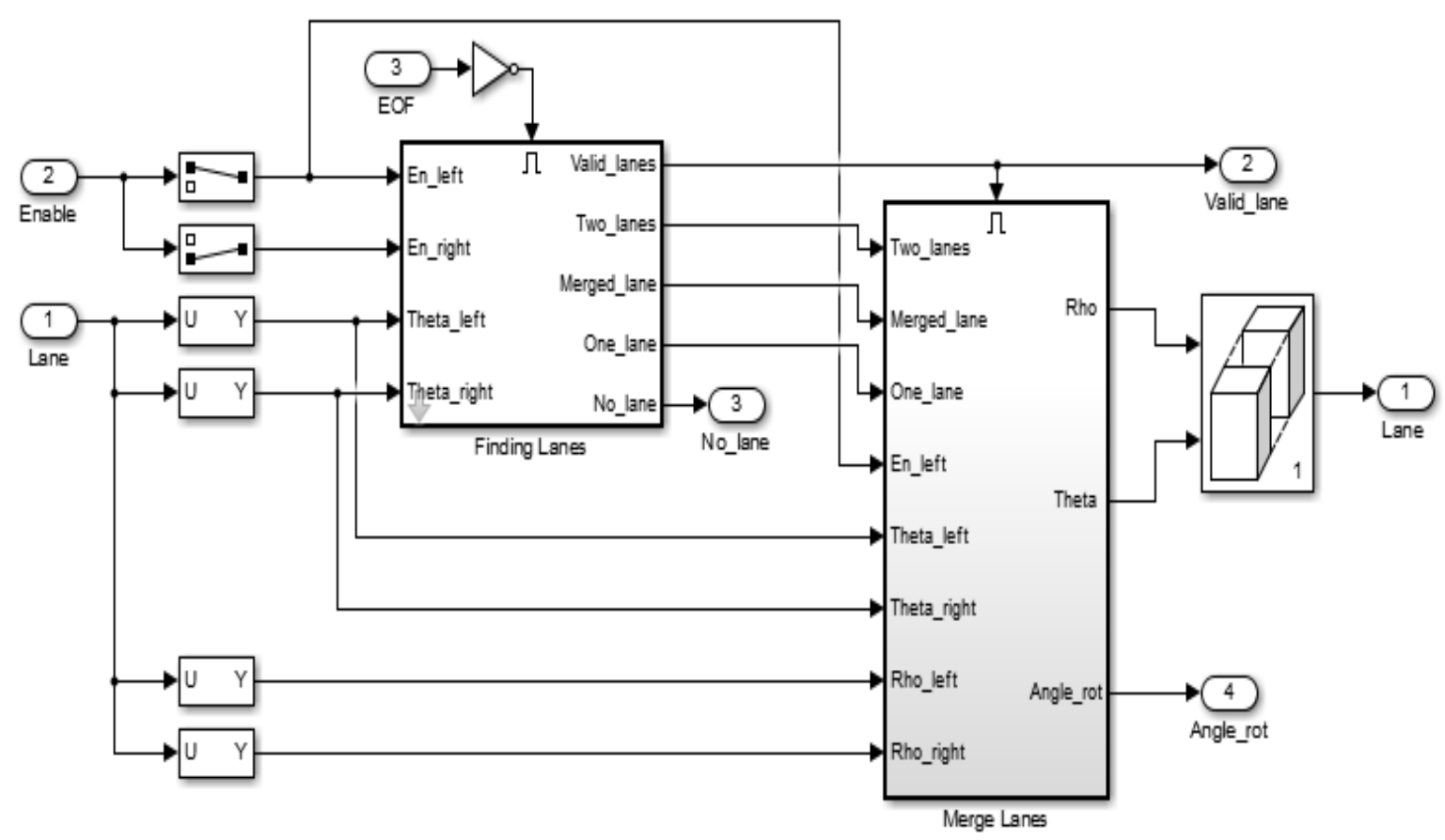

Fig.7.Lane Merging subsystem

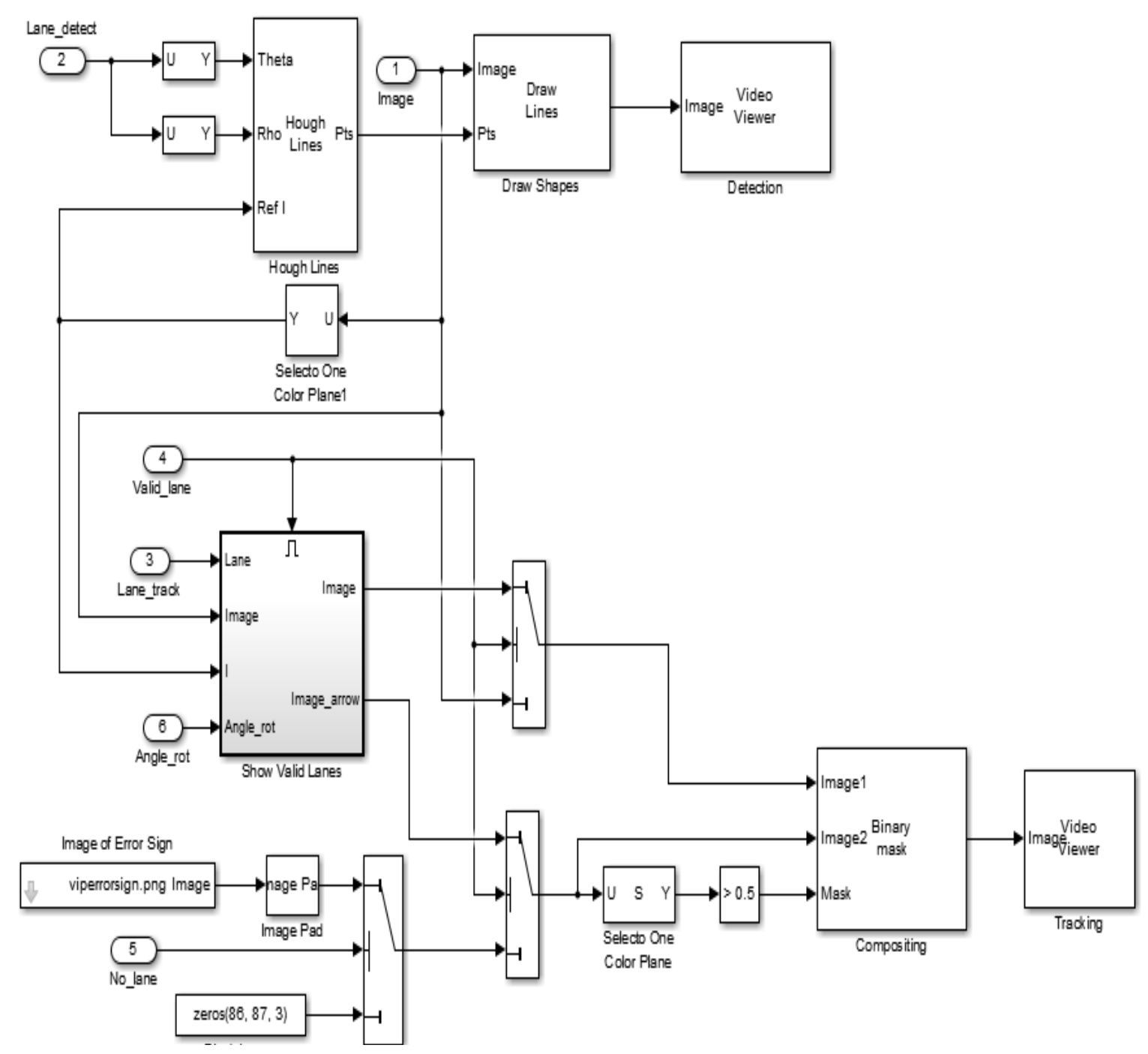

Fig.8. Display subsystem 


\section{Results Analysis}

The suggested algorithm carries out the investigation to describe the right and left edges of road by investigating the image from the video for color behavior changing. The edge pixels and the passing line via number of color pixels has initiated from the center of image bottom. The movement of investigation from upper and right corners of the image as demonstrated in Figure 9.

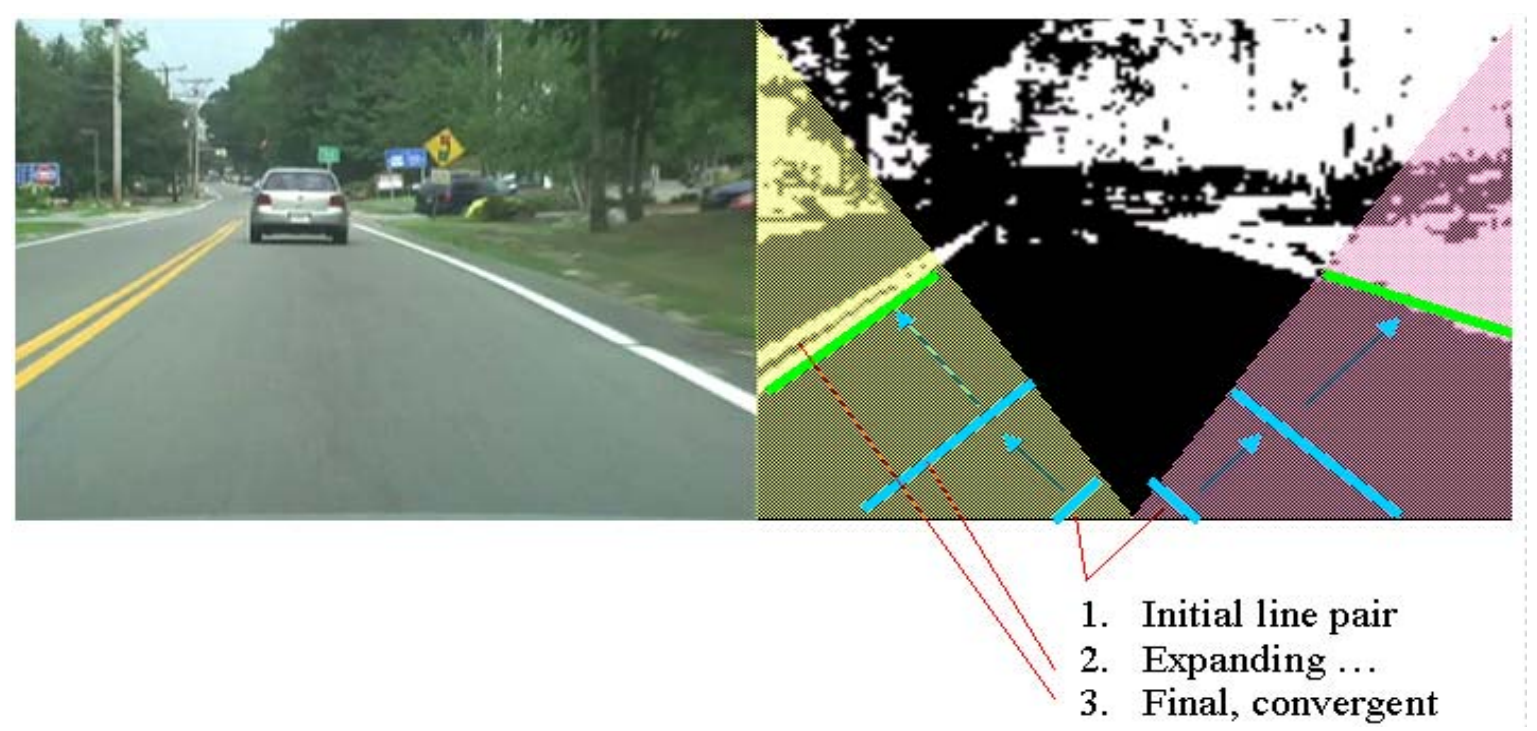

Fig.9. The initial line pair and expanding for final convergent

The algorithms might wait for many frames of valid edge data in case of low quality video sequence and the same process will repeated again to decide when will begin to ignore the side of this images. In the detection window, the road sides will appear and detected in the current frame of video as show in Figure 10.

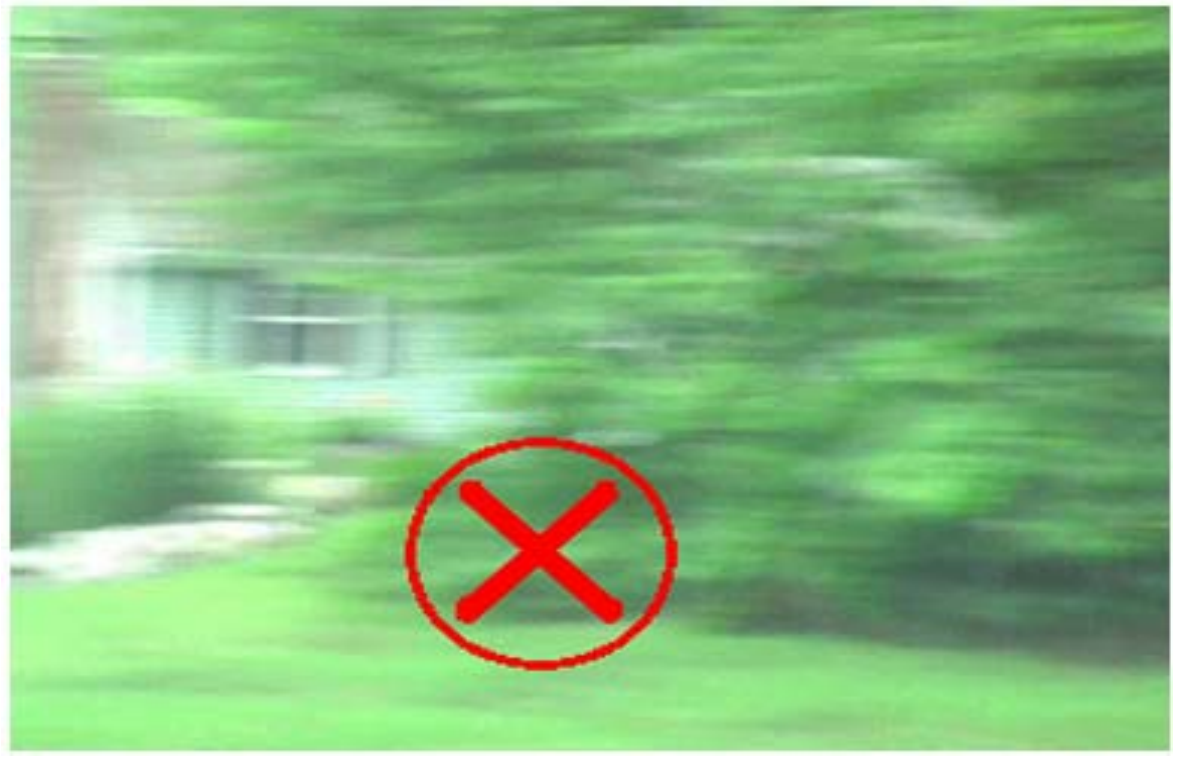

Fig. 10.Error sign in the display window

In case of no road side is showing, the tracking window will show an error symbol as illustrated in Figure 11. In case of only one side of road is appearing, the display will show an arrow in parallel to the road side. The arrow direction is toward the upper region of intersection among the image boundary and road side. When the road of both sides appearing, the display will show an projectile in the center of street in the way which is determined by average of direction of both left and right sides as illustrated in Figure 12. 


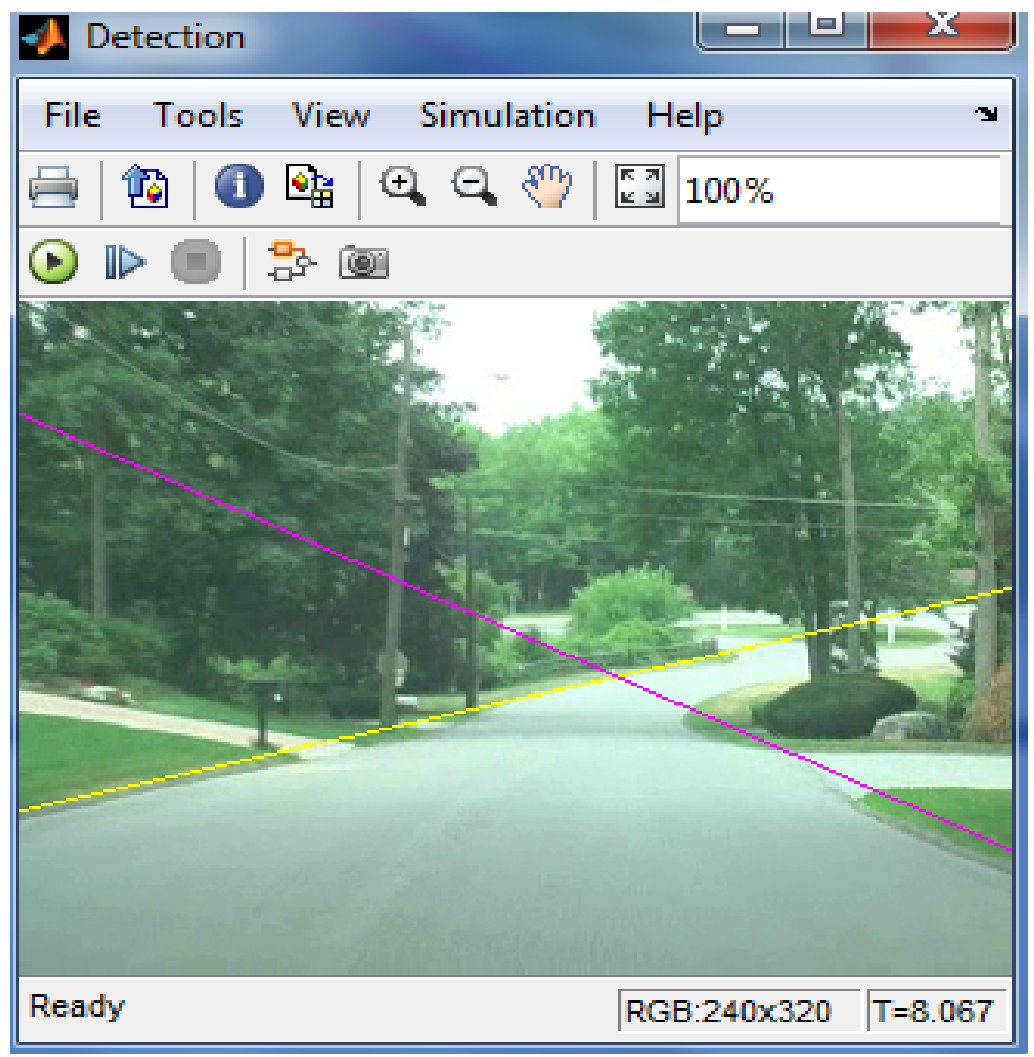

Fig. 11: pathway window

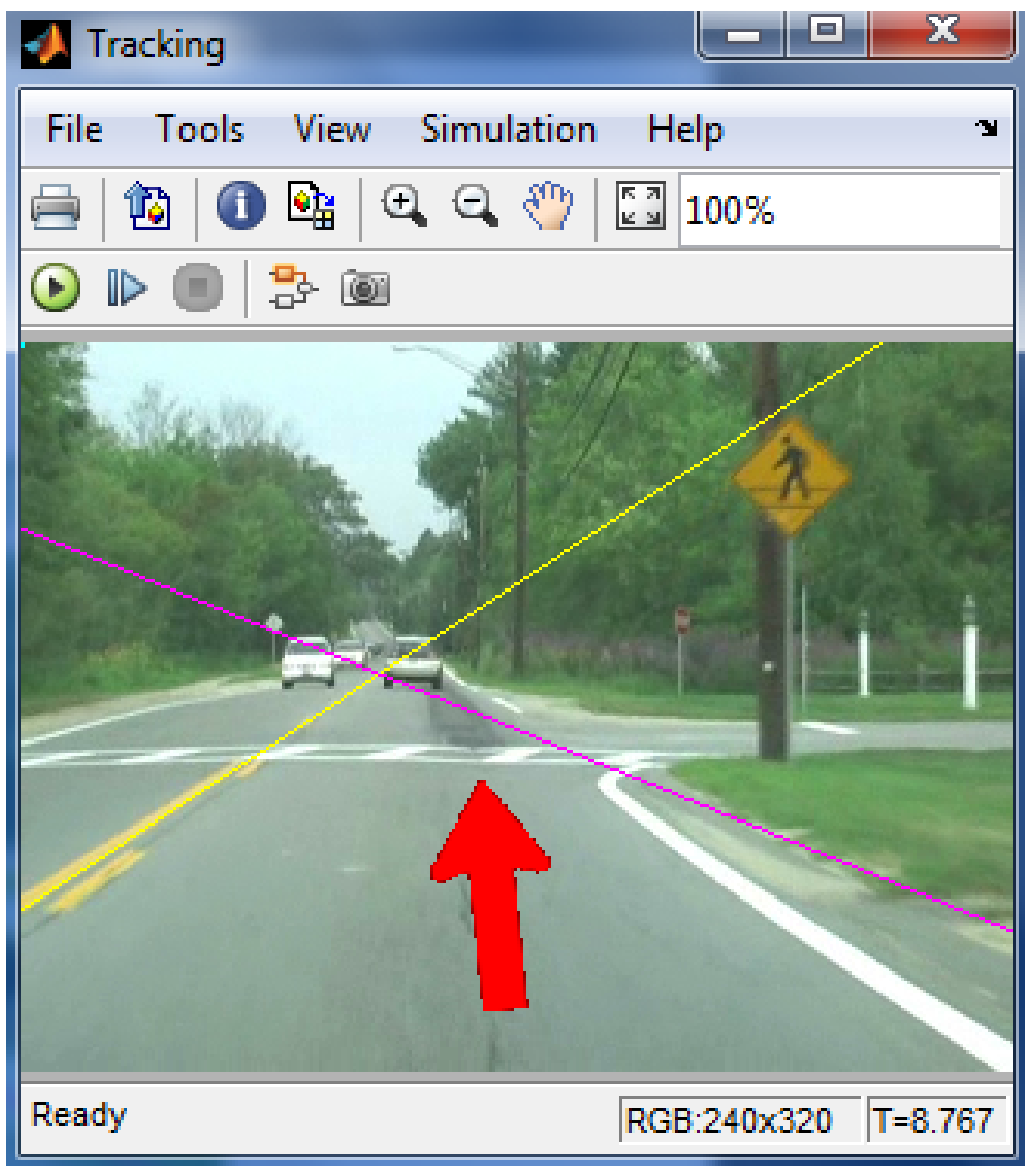

Fig.12. Tracking process window 


\section{CONCLUSION}

This paper introduced efficient algorithm which have been improved for real time tracking and discovery by use color segmentation and region specification. The motion discovery and tracking is performing by environmentof calculationand visualstream techniques. The middle filter is used in the dispensation of image to eliminate the noise through tracking and detection which consider better convolution methods in case of prevent edge and remove the noise from the picture. The suggested algorithm isrealized and tested in MATLAB block set to detect and recognize road sign with accuracy of $85 \%$. This technique needs some development in order to get better accuracy in the range by allowing for different scaling and rotation of road using color image processing combined with shape processing techniques.

\section{REFERENCES}

[1] Jonas Sköld, "Estimating 3D-trajectories from Monocular Video Sequences", Degree Project in Computer Science and Communication, School of Computer Science and Communication, KTH Royal Institute of Technology, Stockholm, Sweden, 2015.

[2] RachnaVerma, “An Efficient Color-Based Object Detection and Tracking in videos” International Journal of Computer Engineering and Applications, vol XI, Issue XI, pp 172-178, Nov 2017.

[3] RachnaVerma, “A Review of Object Detection and Tracking Methods”, International Journal of Advance Engineering and Research Development, vol 4, issue 10, pp569-578, Oct 2017.

[4] C. S. Royden, K. D Moore, “Use Of Speed Cues In The Detection Of Moving Objects By Moving Observers," Vision research, Vol. 59, pp. 17-24, 2012.

[5] Kelson R. T. Aires, Andre M. Santana, Adelardo A. D. Medeiros “Optical Flow Using Color Information”, ACM New York, NY, USA. ISBN 978-1-59593-753-7, 2008.

[6] SepehrAslani, HomayounMahdavi-Nasab, "Optical Flow Based Moving Object Detection and Tracking for Traffic Surveillance", World Academy of Science, Engineering and Technology International Journal of Electrical and Computer Engineering, vol. 7, No:9, 2013.

[7] Fernandes, J.; Neves, J. Using Conical and Spherical Mirrors with Conventional Cameras for $360^{\circ}$ Panorama Views in a Single Image. In Proceedings of the IEEE 3rd International Conference on Mechatronics, Budapest, Hungary, 3-5 July 2006.

[8] Scaramuzza, D. Omnidirectional Vision: From Calibration to Robot Motion Estimation. Ph.D. Thesis, Department of Mechanical and Process Engineering, Swiss Federal Institute of Technology University, ETH Zurich, Switzerland, 2008.

[9] Kotze, B.; Jordaan, G.; Vermaak, H. Development of a Reconfigurable Automatic Guided Vehicle Platform with Omnidirectional Sensing Capabilities. In Proceedings of the 2010 IEEE International Symposium on Industrial Electronics, Bari, Italy, 4-7 October 2010.

[10] Users Guide, Image Acquisitioning Toolbox, Version 1; TheMathworks: Natick, MA, USA, March 2003.

[11] A. Yilmaz, O. Javed, and M. Shah, Object tracking: A survey, ACM Computer Surveys, vol. 38, no. 4,2006.

[12] W. Hu, T. Tan,L. Wang and S. Maybank, A survey of Visual Surveillance of Object Motion andBehaviours, IEEE Trans, vol. 34, no. 3, pp. 334-352, 2004.

[13] X. Wang, Intelligent Multi-Camera Video Surveillance: A review, Pattern Recognition Letters, 2012.

[14] A. D. M. Soto and C. S. Regazzoni, An Overview on Bayesian Tracking for Video Analytics, IEEE,Computer Vision and Image Understanding, vol. 104, no. 1, pp. 90-126, 2010.

[15] H. Yang, L. Shao, F. Zheng, L. Wang and Z. Song, Recent advances and trends in visual tracking: Areview, ELSEVIER, Nerocomputing, vol. 74, pp. 3823-3831, 2011.

[16] L. Li, W. Huang, I. Yu-Hua Gu and Q. Tian, Statistical Modeling of Complex Backgrounds forForeground Object Detection, IEEE Trans, vol. 13, no. 11, pp. 1459-1472, 2004.

[17] M. Liem and D. M. Gavrilla, Multi-person tracking with overlapping cameras in complex, dynamicenvironments, Proc. of the British Machine Vision Conference (BMVC), 2009.

[18] R. Girisha (supervisor: Dr. S. Murali), Some New Methodologies to Track Humans in a SingleEnvironment using Single and Multiple Cameras, Doctoral Thesis, University of Mysore, 2010.

[19] R. Girisha and S. Murali, Tracking humans using novel optical flow algorithm for surveillance videos,Proceedings of the Fourth Annual ACM Bangalore Conference, 2011.

[20] E. Dallalazadeh and D. S. Guru, Feature-based tracking approach for detection of moving vehicle intraffic videos, ACM, Proceedings of the First International Conference on Intelligent InteractiveTechnologies and Multimedia, pp. 254-260, 2010.

[21] K. Nummiaro, E. Koller-Meier, T. Svoboda, D. Roth and L. V. Gool, Color-Based Object Tracking inMulti-Camera Environments, Springer Verlag, DAGM-LNCS, pp. 591-599, 2003.

[22] G. Zhu, Q. Zeng and C. Wang, Efficient edge-based object tracking, ELSEVIER, Pattern Recognition,vol. 39, pp. 2223-2226, 2006.

[23] C. Wang, H. Liu and Y. Gao, Secne-Adaptive Hierarchical Data Association for Multiple ObjectsTracking, IEEE Signal Processing Letters, vol. 21, no. 6, pp. 701, 2014.

[24] A. Martin and J. Martinez, On Collaborative People Detection and Tracking in Complex Scenarios,ELSEVIER, Image and Vision Computing, vol. 30, pp. 345-354, 2012.

[25] M. Kristan, J. Pers, S. Kovacic and A. Leonardis, A local-motion-based probabilistic model for visualtracking, ELSEVIER, Pattern Recognition, vol. 40, pp. 2160-2168, 2009.

[26] A. Gilbert and R. Bowden, Incremental, scalable tracking of objects inter camera, ELSEVIER,Computer Vision and Image Understanding, vol. 111, pp. 43-58, 2008.

[27] I. Haritaoglu, D. Harwood and L. S. Davis, Hydra: Multiple People Detection and tracking usingSilhouettes, IEEE, 1999.

[28] Dong-WookSeo, Hyun-UkChae, Byeong-Woo Kim, Won-Ho Choi and Kang-Hyun Jo, HumanTracking based on Multiple View Homography, Journal of Universal Computer Science, vol. 15, no.13, pp. 2463-2484, 2009.

[29] Zhenghao Xi, Dongmei Xu, Wanqing Song, Yang Zheng, A* algorithm with dynamic weights formultiple object tracking in video sequence, Optik - International Journal for Light and ElectronOptics, Volume 126, Issue 20, October 2015

[30] HamidrezaJahandide, Kamal Mohamedpour, Hamid AbrishamiMoghaddam, A hybrid motion andappearance prediction model for robust visual object tracking, Pattern Recognition Letters, Volume33, Issue 16, 1 December 2012

[31] A. Heili, C. Chen and Jean-Marc Odobez, Detection-Based Multi-Human Tracking Using a CRFModel, Proceedings of IEEE ICCV, 2011.

[32] R. V. Babu, P. Perez and P. Bouthemy, Robust tracking with motion estimation and local Kernel-basedcolor modeling, ELSEVIER, Image and Vision Computing, vol. 25, pp. 1205-1216, 2007. 
[33] N. Thome, D. Merad and S. Miguet, Learning articulated appearance models for tracking humans: Aspectral graph matching approach, ELSEVIER, Image Communication, vol. 23, pp. 769-787, 2008.

[34] I. Ali and M. N. Dailey, Multiple Human Tracking in High-Density Crowds, ELSEVIER, Image andVision Computing,(in press) ,2012.

[35] A. Yamashita, Y. Ito, T. Kaneko and H. Asama, Human Tracking with Multiple Cameras Based onFace Detection and Mean Shift, Proceeding of IEEE International Conference on Robotics andBiometrics, pp. 1664-1671, 2011. 